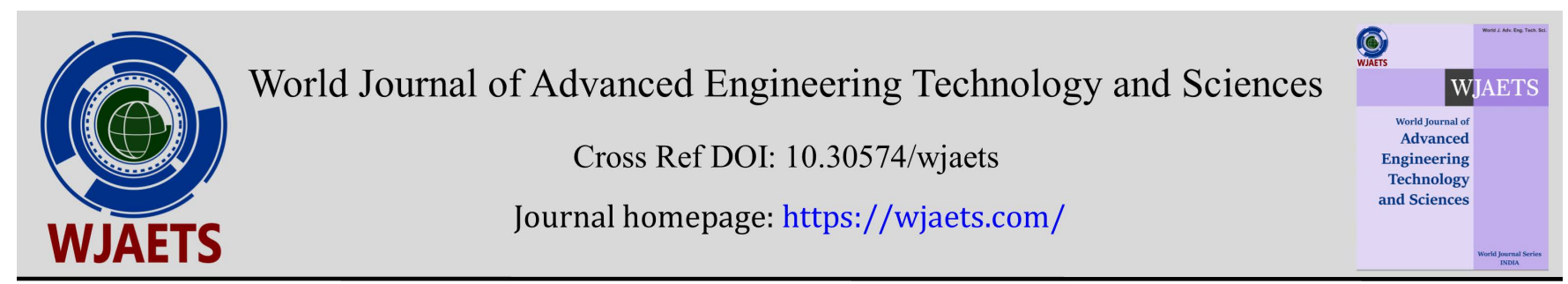

(RESEARCh ARTicle)

\title{
Improvement of weld heat affected zone (HAZ) using Taguchi method
}

\author{
Nweze Stephanie* and Achebo J \\ Department of Production Engineering University of Benin, Benin City, Nigeria.
}

World Journal of Advanced Engineering Technology and Sciences, 2021, 02(01), 027-033

Publication history: Received on 30 November 2021; revised on 10 January 2021; accepted on 12 January 2021

Article DOI: https://doi.org/10.30574/wjaets.2021.2.1.0020

\begin{abstract}
Heat Affected Zone (HAZ) is the area on a weldment mostly affected by the intensity of the applied heat without melting. This area mostly deteriorates faster due to microstructural changes that occur due to the intensity of the applied heat during welding operations. Weld structural failures can be catastrophic and unpredictable at times. When there is no loss of human life involved, damages as a result of weld failure usually takes more time and cost more to repair/replace. For these reasons, a weld with good quality integrity cannot be over-emphasized. The larger the HAZ, the wider the area with microstructural alteration, the lesser the quality integrity of the weldment. In this study, extensive research was conducted to reduce weld HAZ using the Taguchi method. This method makes use of signal to noise ratio of responses to achieve optimality. From applying this model, it was observed that the best input parameters, that improved the weld quality was achieved. These input parameters were current of $120 \mathrm{~A}$, voltage of $10 \mathrm{~V}$ and gas flow rate of $144 \mathrm{~L} / \mathrm{min}$. From the analysis of variance (ANOVA), it was found that current contributed about $9.58 \%$ to the weld quality, which was the most influential process parameters. The confirmation test done, shows that the weldment produced by using the optimum process parameters had an improvement of $1.37 \mathrm{db}$ and $0.88 \mathrm{~mm}$ reduction in $\mathrm{HAZ}$, over the weldment made by existing parameters.
\end{abstract}

Keywords: Heat Affected Zone (HAZ); Mild steel; Gas flow rate (GFR); Signal to noise ratio (S/N)

\section{Introduction}

In welding operations, keeping the Heat Affected Zone (HAZ) at its lowest has been the major focus of most researches, since smaller HAZ helps improve the quality integrity of the weldment as only a smaller area is affected by microstructural changes resulting from the welding activities. Weld failure has caused damages to innumerable properties, and in some cases resulting to loss of human life. Most of these failures occur at welded joints or HAZ. In order to improve on the quality of the welded joint, input process parameters needs to be optimized.

Intensive arc heat as applied to welding process causes distortion in the microstructural arrangements of the metal grains contained in the weldment (Alam and Khan, 2012). Depending on the level of the heat treatment given by the intensive arc heat, the welded joint is either tough or has reduced strength factor. Low strength weldment is susceptible to failure especially when the weldment is designed to carry heavy loads. When the strength of a weldment is less than that of the parent metal, the load bearing capacity of that weldment is assumed to be low (Achebo, 2012). Patil and Waghmare (2013) were of the opinion that the manufacturing industries could reduce weld failure if proper control of input process parameters is achieved. This would help obtain a good welded joint with the required weld quality.

Achebo (2011) and Kishore et al. (2010) observed that steel alloys are susceptible to distortion due to their high coefficient of thermal expansion. In some cases, certain steel alloys are quite prone to cracking and reduced corrosion resistance. Pal (2015) in his study optimized the input process parameters used for welding some medium carbon steel

\footnotetext{
${ }^{*}$ Corresponding author: Nweze Stephanie

Department of Production Engineering University of Benin, Benin City, Nigeria.
}

Copyright (c) 2021 Author(s) retain the copyright of this article. This article is published under the terms of the Creative Commons Attribution Liscense 4.0. 
slabs required to improve their tensile properties. The ultimate tensile strength of the welded joint and the elongation produced during the tensile test were considered as vital responses needed for improving weldment strength and quality. From his study, it was observed that welding current and welding speed are major input parameters that influenced the ultimate tensile strength of the weldment. Meshram and Pohokar (2013) worked on the optimization of input process parameters of gas metal arc welding to improve the quality of weld bead geometry by applying the grey based Taguchi method. In their case, stainless steel (AIS1410) welded joint was the material used for the study. Since distorted areas in welded plates are susceptible to cracking, minimizing such areas would help to increase the quality of the weldments. Therefore, localization of the welding process in a controlled manner would help reduce the heat affected zone of the weldment.

This study is aimed to reduce the heat affected zone of weldment by determining a combination of process parameters that would aid in achieving that.

\section{Material and methods}

Gas Metal Arc Welding (GMAW) process was used to join nine (9) samples of $10 \mathrm{~mm}$ mild steel plates, power saw was used to cut the plates into the required dimensions of $50 \mathrm{~mm}$ by $100 \mathrm{~mm}$. The input parameters used for this study are current, voltage and gas flow rate presented in Table 1. The welding machines contain the welding gun, shielding gas consisting of $100 \%$ argon. A $1.6 \mathrm{~mm}$ consumable wire electrode of AWS classification ER70S-3 was used. The heat affected zone was the targeted response of the welded sample.

Table 1 Range of Process Parameters

\begin{tabular}{llll}
\hline Factor's & \multicolumn{3}{c}{ levels } \\
& 1 & 2 & 3 \\
\hline A. Voltage, $V$ & 10 & 12 & 14 \\
B. Current, A & 120 & 140 & 160 \\
C. Gas Flow rate, Litre/min & 14 & 15 & 16 \\
\hline
\end{tabular}

The following steps were followed to achieve optimum results in the course of this study

- The Taguchi orthogonal array was used to develop the experimental matrix design.

- The Experimental test was carried out according to each input process parameters of the experimental run.

- The signal to noise ratio is determined for each responses.

- Optimum signal to noise ratio should be selected.

- The analysis of variance is calculated to determine the contribution of each input process parameters to the quality improvement of the weldment.

- A confirmation test is done to validate the potency and superiority of the optimum welding parameters over the existing process parameters.

\section{Results}

Table 2 shows the orthogonal matrix layout for the nine weld samples and heat affected zone measurements.

Note that to remove the negativity of the S/N ratio of HAZ values of the weldments, the measured HAZ values was normalized for ease of calculation.

Applying the smaller the better methodology by using Eq. (1)

Signal to Noise ratio for the smaller the better function is

$\eta=-10 \log _{10}\left(\frac{1}{n} \sum_{i=1}^{n} y_{i}^{2}\right)$

Where, $\mathrm{n}$ is the sample size and $\mathrm{y}$ is the mean weld HAZ width in mm 
For experiment 1,

$\eta=-10 \log _{10}\left(\frac{1}{1} \sum_{i=1}^{n} 0.1049^{2}\right)=19.5845$

Experiment 2-9 are obtained using same procedure as in experiment 1, Table 3 present summaries of S/N ratio values obtained from each experiment

Table 2 Orthogonal Matrix Layout and Heat Affected Zones (HAZ) Measurements of corresponding Weldments

\begin{tabular}{c|ccc|cc}
\hline Experiment & current & voltage & Gas flow rate & HAZ width & Normalized \\
rums & $I$ & $V$ & L/min & mm & HAZ width, mm \\
\hline 1 & 1 & 1 & 1 & 1.25 & 0.1049 \\
2 & 1 & 2 & 2 & 1.62 & 0.1359 \\
3 & 1 & 3 & 3 & 0.98 & 0.0822 \\
4 & 2 & 1 & 3 & 0.70 & 0.0587 \\
5 & 2 & 2 & 1 & 1.85 & 0.1552 \\
6 & 2 & 3 & 2 & 1.58 & 0.1326 \\
7 & 3 & 1 & 2 & 1.36 & 0.1141 \\
8 & 3 & 2 & 3 & 1.18 & 0.0990 \\
9 & 3 & 3 & 1 & 1.40 & 0.1174 \\
\hline$\sum$ & \multicolumn{5}{r}{}
\end{tabular}

Table $3 \mathrm{~S} / \mathrm{N}$ ratio for all the experiments

\begin{tabular}{|l|l|l|l|l|l|}
\hline Experiment & S/N ratio & Experiment & S/N ratio & Experiment & S/N ratio \\
\hline 1 & 19.5845 & 4 & 24.6272 & 7 & 18.8543 \\
\hline 2 & 17.3356 & 5 & 16.1822 & 8 & 20.0873 \\
\hline 3 & 21.7026 & 6 & 17.5491 & 9 & 18.6066 \\
\hline
\end{tabular}

Athreya and Venkatesh (2012) used the smaller the better type of Taguchi method and wrote that the process parameters with the highest $\mathrm{S} / \mathrm{N}$ ratio is regarded as the optimum. In this study, the highest S/N ratio is 24.6272 obtained from conducting experimental run 4.

The $\mathrm{S} / \mathrm{N}$ ratio for the individual control factors as extracted from Table 3 . The average $\mathrm{S} / \mathrm{N}$ ratios for the process parameters are shown in Table 4.

Table 4 Sum and Average S/N ratio for each Process Parameter

\begin{tabular}{|l|l|l|l|l|}
\hline Level & A. Current & B. Voltage & $\begin{array}{l}\text { C. Gas } \\
\text { Flow rate }\end{array}$ & $\begin{array}{l}\text { Total Average } \\
\text { of S/N ratio }\end{array}$ \\
\hline & $\begin{array}{l}\text { Average of } \\
\text { S/N ratio }\end{array}$ & $\begin{array}{l}\text { Average of } \\
\text { S/N ratio }\end{array}$ & $\begin{array}{l}\text { Average of } \\
\text { S/N ratio }\end{array}$ & \multirow{2}{*}{19.3921} \\
\cline { 1 - 4 } 1 & $19.5409 *$ & $21.0220 *$ & 19.0736 & \\
\cline { 1 - 4 } 2 & 19.4528 & 17.8684 & $20.1898 *$ & \\
\cline { 1 - 4 } 3 & 19.1827 & 19.2861 & 18.9130 & \\
\hline
\end{tabular}

*signifies the optimum level based on the smaller-the-better criterion. Athreya and Venkatesh (2012) calculated the surface roughness of lathe facing operation by using the smaller the better type of Taguchi method and selected the factor levels corresponding to the highest $\mathrm{S} / \mathrm{N}$ ratio as the optimum process parameters. From Table 4, the optimum level corresponding to the highest S/N Ratio is $\mathbf{A}_{\mathbf{1}}-\mathbf{B}_{\mathbf{1}}-\mathbf{C}_{\mathbf{2}}$ 
Plots of S/N ratio vs current, voltage and gas flow rate is presented in Figures 1 -3.

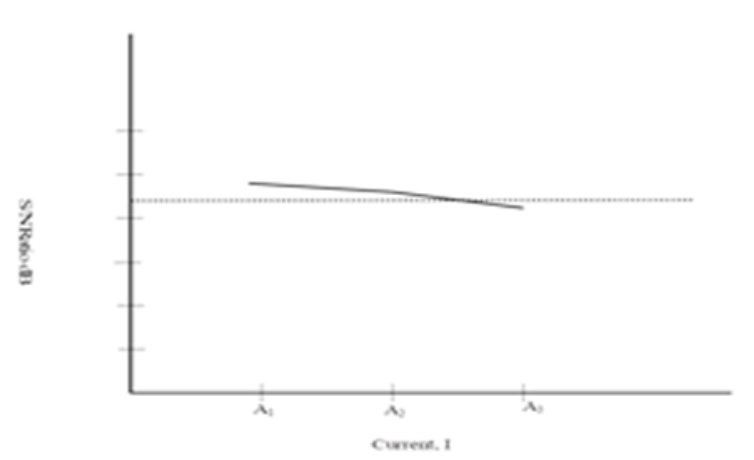

Fig 1: S/N Ratio vs Current

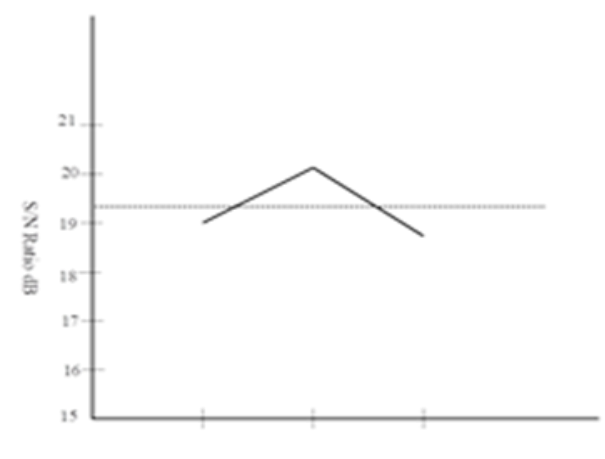

Cas Eoo rats, Limin

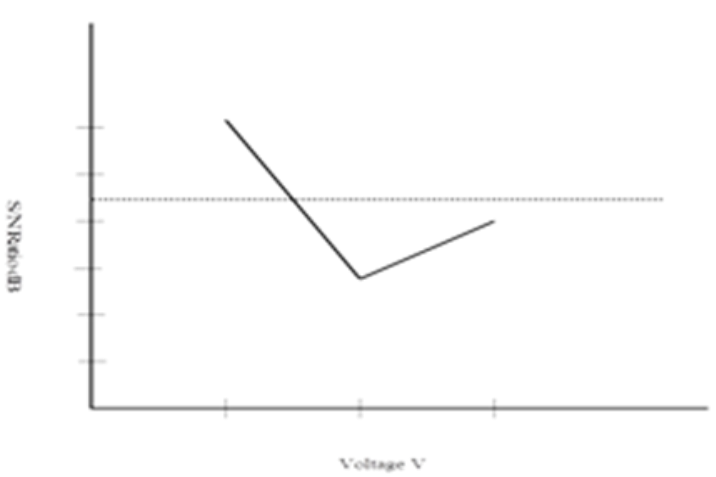

Fig. $2 \mathrm{~S} / \mathrm{N}$ Ratio vs Voltage

Fig 3: S/N Ratio vs Gas flow rate

Table 5 shows the analysis of variance tabulation, with Eq. (2), (3) and (4) used to calculate the sum of square, percentage contribution and F-ratio.

Table 5 Analysis of Variance (ANOVA)

\begin{tabular}{|l|l|l|l|l|l|l|}
\hline Parameter & $\begin{array}{l}\text { Process } \\
\text { Parameter }\end{array}$ & $\begin{array}{l}\text { Degree of } \\
\text { Freedom }\end{array}$ & $\begin{array}{l}\text { Sum of } \\
\text { Squares }\end{array}$ & Variance & F-ratio & $\begin{array}{l}\text { Contribution } \\
\text { (\%) }\end{array}$ \\
\hline A & Voltage, V & 2 & 0.0697 & 0.0349 & 0.0015 & 0.1338 \\
\hline B & Current, A & 2 & 4.9895 & 2.4948 & 0.1083 & 9.5767 \\
\hline C & $\begin{array}{l}\text { Gas flow rate, } \\
\text { Litre/min }\end{array}$ & 2 & 0.9673 & 0.4837 & 0.0210 & 1.8566 \\
\hline Error & & 2 & 46.0739 & 23.0370 & - & 88.4329 \\
\hline Total & & 8 & 52.1004 & - & - & 100 \\
\hline
\end{tabular}

Sum of square, $S_{i}^{2}=\sum\left(y_{i}^{2}\right)-\frac{\left(\sum y_{i}\right)^{2}}{n}$

Where $\mathrm{n}$ is the number of test conducted (Achebo, 2012)

$\%$ Contribution $=\frac{S_{i}^{2}}{S_{T}^{2}} \times 100 \%($ Achebo, 2012$)$ 
F-ratio $=\frac{\text { Variance }_{i}}{\text { Variance }_{\text {error }}}($ Achebo, 2012)

\subsubsection{Confirmation Test}

The confirmation test is done to validate the results obtained by using the signal to noise ratio and to demonstrate the potency of the optimum process parameters. The Confirmation test is done by using Eq (5)

$\eta=\eta_{m}+\sum_{i=1}^{n}\left(\bar{\eta}-\eta_{m}\right)$

Where $\eta_{m}$ is the total mean of $\mathrm{S} / \mathrm{N}$ ratio; $\bar{\eta}$ is the mean of $\mathrm{S} / \mathrm{N}$ ratio at the optimal level and $\mathrm{n}$ is the number of main welding parameters.

The optimal parameters are $\mathrm{A}_{1}-\mathrm{B}_{1}-\mathrm{C}_{2}$ and the corresponding $\mathrm{S} / \mathrm{N}$ ratios are $19.5409,21.0220$ and 20.1898 respectively.

The total mean of $\mathrm{S} / \mathrm{N}$ ratio, $\eta_{m}=19.3921$

Therefore applying Eq (5), $\eta=21.9685$

The existing process parameters in use for welding processes were $\mathrm{A}_{2}-\mathrm{B}_{1}-\mathrm{C}_{3}$

By applying Eq (5), Its $\mathrm{S} / \mathrm{N}$ ratio was calculated to be $\eta=20.6036$

Table 6 shows a summary of the confirmation test results

Table 6 Confirmation Test Results

\begin{tabular}{llll}
\hline & $\begin{array}{l}\text { Existing } \\
\text { Process } \\
\text { Parameters }\end{array}$ & $\begin{array}{l}\text { Optimum } \\
\text { Process } \\
\text { Parameters }\end{array}$ & $\begin{array}{l}\text { Improvement } \\
\text { in S/N ratio and } \\
\mathrm{HAZ} \text { width }\end{array}$ \\
\hline Process Parameters & $\mathrm{A}_{2}-B_{1}-C_{3}$ & $\mathrm{~A}_{1}-B_{1}-C_{2}$ & \\
$\mathrm{HAZ}$ width (mm) & 1.42 & 0.54 & 0.88 \\
S/N dB & 20.6036 & 21.9685 & 1.3649 \\
\hline
\end{tabular}

\section{Discussion}

In this study, the Taguchi optimization method was used to select the weldment with the best input process parameters and HAZ width. Table 1 contains the process parameters which include, the welding current denoted as A, voltage denoted as B and gas flow rate denoted as C. Table 2, contains the output parameter which is the HAZ width. The HAZ expresses the extent to which the localized arc heat affects and distorts the orientation of the micro-structural arrangement of the parent metal, which is the piece of metal to be joined.

A good parent metal is highly dense with fine grained microstructural arrangement. When joined by wielding, as the arc heat increase and welding gas decreases, the solid filler metal transit into molten metal and fills the gap in-between the parent metal, the shielding gas which tend to shield the welding environment may not be sufficient enough to protect the environment from air infiltration. Sufficient air inter mixing with the molten metal may be trapped in the weldment during the cooling process. This is because molten metal cools very fast, therefore before the evacuation of the air from the molten metal as it is cooling very fast, sufficient air maybe left inside the weldment. The leftover air is as a result of the fact that the rate of air evaporation from the hot molten metal is lower than the cooling rate of the weldment. Therefore the leftover air, if sufficient enough could oxidize the micro structural grains of the weldment or weldmetal, 
which transforms these microstructures into macrostructures due to oxidization. Macro-structural grains have heterogeneous shape, which contains misaligned grains with irregular shape and create voids inside the weldment. At this point sufficient load applied could cause metal indentation which lowers the quality of the weldment, because weldment of good quality are dense in nature and the grains are closely and tightly packed together.

The above explanation expresses the reason why the lower or smaller the HAZ width the better the quality of the weldment. In using the Taguchi method to analyse the output parameter, the smaller the heat affected zone width, the better the quality of the weldment and the higher the signal-to-noise ratio, was used in this study.Table 1 shows the input parameters alongside with their levels. The levels is divided into three parts, the low, medium and high. Applying the smaller the better quality criteria, the signal to noise ratio of the output processes parameter was obtained. The higher the signal to noise the better the quality characteristics of the weldment. In this study, weldment 4 has the highest signal to noise ratio of 24.6272. The signal to noise ratio of the individual input process parameter at the different levels were computed and averaged. The average values are recorded in Table 4.From Table 4, under each process parameter, the level with the highest signal to noise ratio is selected that is, for current 19.5409 , for voltage 21.0220 and For gas rate 20.1898 were selected from the obtained values, the optimum process parameters becomes A1,B1,C2. Figures 1 shows the effect of the signal to noise ratio on the welding current. It is shown that A1 and A2 are above the mean signal ratio, which make them significant that is, the noise of variation in the welding process can be controlled, but since $\mathrm{A} 1$ has the least noise, it is considered the optimum value. A3 is found to be below the mean signal to noise ratio, this indicates that it contains a lot of noise which would alter the quality of the weldment by allowing the infiltration of Impurities into the molten weldment. Figure2 shows the effect of signal to noise ratio on the welding voltage. From Figure 2 it can be seen that B1 is above the mean signal to noise ratio, making it has less controllable amount of noise in the welding process whereas, B2 and B $\neg 3$ are below the mean signal to noise ratio, therefore they contain uncontrollable amount of noise in the entire welding process. However B2 is found to have a higher noise level than B3. Figure 3 shows the effect of the signal to noise ratio on the gas flow rate. $\mathrm{C} 1$ falls below the mean signal to noise ratio, which makes it has uncontrollable noise level whereas $\mathrm{C} 2$ is seen to be above the mean signal to noise ratio. This indicates that the noise level is controllable and highly minimized. C3 is below the mean signal to noise ratio and it is found to have more uncontrollable noise level.

The analysis of variance was computed to determine the level of contribution of each of the process parameter to the quality level of the weldment. Table 5 shows the computation process of analysis of variance. From Table 5 , it can be seen that current contributed most having a total of $9.58 \%$ of the quality level of the weldment, followed by the welding speed, with a value of $1.86 \%$ and voltage being the least with a contribution of $0.13 \%$

A confirmation test was carried out to validate the inference that A1,B1,C2 is the optimum process parameters. The signal to noise ratio of the existing process parameters of A2 B1, C3 was determined to be 20.60dB, whereas, the optimum welding process has a signal to noise ratio of $21.97 \mathrm{~dB}$. This shows that there is an improvement of $1.37 \mathrm{~dB}$ the optimum process parameters has over the existing one. Also the heat affected zones (HAZ) of the weldment produced by the welding operation made by using the optimum process parameters has $0.88 \mathrm{~mm}$ less than the heat affected zone obtained from the weldment made by using the existing process parameters.

\section{Conclusion}

This study was aimed at determining the optimum welding parameters using the Taguchi method. The Taguchi method was able to develop new welding process parameters used by the welders. The newly developed process parameters has a higher signal to noise ratio and less heat affected zone than the ones produced by the existing process parameters.

Optimal process parameters are an important factor to be considered because it can be used to evaluate the quality of the weldment.

\section{Compliance with ethical standards}

\section{Acknowledgments}

We acknowledge the department of Production, University of Benin and its workshop for their support during this research.

\section{Disclosure of conflict of interest}

There is no conflict of interest as regards this work 


\section{References}

[1] Alam S And Khan MI. Prediction Of Weld Bead Reinforcement Height For Steel Using Saw Process Parameters, International Journal Of Applied Engineering Research. Issn 0973-4562, 6(152011): 1857-1871.

[2] Achebo JI. Optimization of Fluence Energy in Relation to Weld Properties based on Vogel Approximation Method. delivered at the 2012 World Congress on Engineering: International Conference on Mechanical Engineering, Imperial College, London. July $4-62012$.

[3] Patil SR and Waghmare CA. Optimization of MIG Welding parameters for improving strength of welded joints. International Journal of Advanced Engineering research and studies. 2013; 11(IV): 14-16.

[4] Kishore K, Gopal Krishria PV, Veladri K, Ali syed Qasim. Analysis of Defects in Gas shielded Arc Welding of AISI 1040 steel using Taguchi method. ARPN Journal of Engineering and Applied sciences. 2010; 5(1): 37-41.

[5] Achebo JI. Optimization of GMAW Protocols and Parameters for Improving Weld Strength Quality' delivered at the 2011 World Congress on Engineering: International Conference of Manufacturing Engineering and Engineering Management, Imperial College, London. July 6 - 7, 2011.

[6] Pal A. MIG Welding Parameteric optimization using Taguchi's orthogonal Array and Analysis of variance. International Journal of Research review in Engineering Science and Technology. 2015; 4(1): 211-217.

[7] Meshram SR, Pohokar NS. Optimization of process parameters of Gas metal Arc welding to improve Quality of weld bead geometry. International Journal of Engineering business and Enterprise Applications. 2013; 46-52.

[8] Athreya, S. and Venkatesh, Y.D. (2012) Application of Taguchi Method for Optimization of Process Parameters in Improving the Surface Roughness of Lathe Facing Operation. International Refereed Journal of Engineering and Science (IRJES), 1, 13-19. 